\section{Conveying that sinking feeling}

Andrew Watson

THE great ocean conveyor is the name coined by Wallace Broecker for the massive circulation cell which transports surface water north through the Atlantic until, in the Labrador and GreenlandNorwegian seas, it becomes sufficiently dense to sink and begin the journey southward again. Western Europeans have reason to be grateful for this circulation, because the heat it transports keeps us warm in winter and saves us from the rigours of a Muscovite climate. Now Broecker and Tsung-Hung Peng show on page 587 of this issue ${ }^{1}$ that the ocean conveyor (less picturesquely known as the Atlantic thermohaline circulation) also transports a substantial amount of carbon dioxide of atmospheric origin between Northern and Southern Hemispheres. The implication is that, before the industrial revolution changed everything, the atmosphere must have transported an equal flux in the opposite direction, from south to north. Carbon dioxide concentrations must therefore have once been higher in the Southern Hemisphere than in the north. This in turn bears upon the current controversy over the size and location of the sinks on land or at sea for fossil-fuel $\mathrm{CO}_{2}$.

Broecker is an acknowledged grand master of chemical oceanography, and his latest paper with Peng demonstrates well his skill at re-interpreting oceanographic data. Starting with widely available measurements on the distribution in the sea of phosphate nutrient and alkalinity (the excess of positive charge balanced by carbonate and bicarbonate ions), they perform simple manipulations to take account of the known effects other than exchange with the atmosphere which would be expected to alter the carbon content. They thereby produce estimates of the concentration of carbon that the water would have contained had it been isolated from the atmosphere. The difference between this 'expected' carbon content and what is actually measured is the amount that has been taken up from, or lost to, the atmosphere. Another quick correction for the amount taken up since the industrial revolution and they arrive at estimates for the pre-industrial ocean surface uptake.

The authors' results show a large excess of $\mathrm{CO}_{2}$ of atmospheric origin in the North Atlantic compared with the Antarctic. Multiplying the difference by the water flux, the rate at which the conveyor turns, they obtain the flux of carbon, 0.6 gigatonnes (Gt) a year, which the Atlantic transported from north to south before 1850 . It all sounds so reasonable that one wonders why it was not done a decade ago. pheric $\mathrm{CO}_{2}$ has therefore changed sign during recent times. Whereas previously $\mathrm{CO}_{2}$ concentrations were higher in the south owing to this ocean transport, the north now has the higher values and the gradient appears still to be increasing ${ }^{2,3}$. Broecker and Peng also say that their result eliminates the need to challenge the earlier estimates of the magnitude of the ocean uptake and the need to postulate a large mid-latitude sink on the land surface, but the reasoning behind this statement is rather more obscure.

Until 1990, the accepted view was that the oceans were the main natural sink for anthropogenic $\mathrm{CO}_{2}$ (the excess produced by fossil-fuel burning and tropical
The north-south gradient of atmoswith the air-sea flux industrially, the net deforestation). Ocean uptake was thought to be about $2 \mathrm{Gt}$ of carbon per year, a figure obtained by 'box-diffusion' analyses calibrated against observations of the uptake of ${ }^{14} \mathrm{C}$ generated in the atmosphere by nuclear testing ${ }^{4,5}$. A more detailed ocean general circulation model has recently given essentially the same result ${ }^{6}$.

Confidence in the models was severely shaken with the publication in 1990 of a study by Pieter Tans and colleagues? This combined an analysis of the distribution of $\mathrm{CO}_{2}$ in the atmosphere with the first detailed attempt to calculate fluxes into the sea using measurements of sea-surface $\mathrm{CO}_{2}$. Tans et al. found that the observed small north-south gradient of $\mathrm{CO}_{2}$ in the atmosphere pointed to a natural sink in the Northern Hemisphere which must exceed that in the south by about $2 \mathrm{Gt} \mathrm{yr}^{-1}$, while the total sink must add up to at least 2.3 and

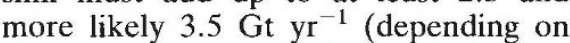
the size of the poorly known deforesta-

\title{
Balancing the carbon budget
}

In related work, Sarmiento and Sundquist point out on page 589 of this issue ${ }^{11}$ that estimates of global air-sea $\mathrm{CO}_{2}$ fluxes based on tracer-calibrated models of ocean mixing are not directly comparable calculations performed by Tans et al. ${ }^{7}$. The models estimate only the additional $\mathrm{CO}_{2}$ now being taken up as a result of the additional atmospheric concentration due to industrialIzation. By contrast, the air-sea exchange approach refers to the actual present-day flux. The two calculations would be expected to agree only if, pre-

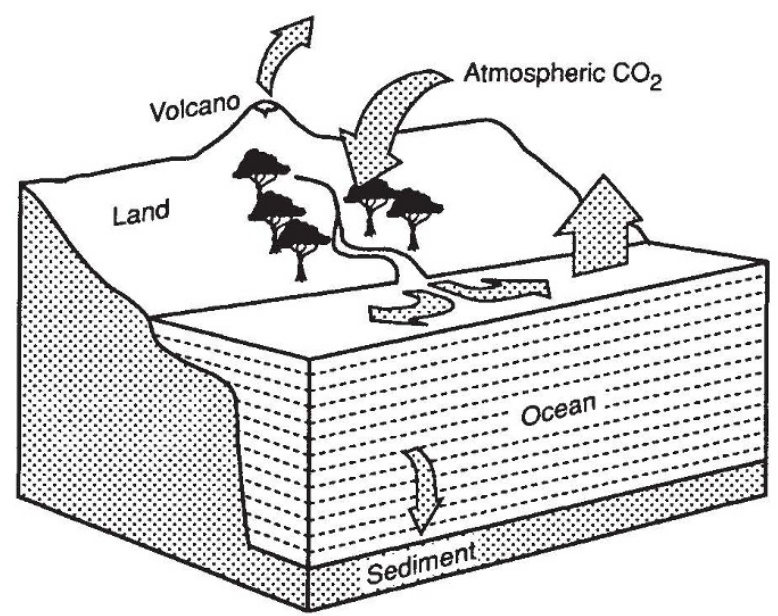

The pre-industrial steady-state carbon cycle: to balance the flux of carbon coming down rivers, there must have been a $\mathrm{CO}_{2}$ flux of the order of 0.5 gigatonnes of carbon per year from ocean to atmosphere and from atmosphere to land. Volcanic activity and sedimentation fluxes provide smaller net inputs and outputs to the system.

transfer from air to sea was zero. But in fact, at that time there must have been a net flux from the oceans to the atmosphere. This is evident because rivers transport a substantial amount of carbon (in the form of organics and bicarbonate) from land to sea. Most of this carbon has come rather directly from the atmosphere or the land biota, and therefore a roughly equivalent flux must be postulated from the sea to the air to complete the steady state. After making corrections to this simple picture to account for carbon lost to sediments or produced by volcanic and metamorphic activity, Sarmiento and Sundquist arrive at a figure of $0.4-0.7$ gigatonnes of carbon per year as the probable pre-industrial flux of $\mathrm{CO}_{2}$ from the oceans to the atmosphere. This is the amount that absorption calculated from a tracer-calibrated ocean model might be expected to exceed the true present-day air-to-sea flux. In their 'new budget', Sarmiento and Sundquist also include a rough estimate of another correction, concerned with the ocean surface 'skin-temperature devlation', which ralses the $\mathrm{CO}_{2}$ concentration at the Interface (and which they kindly attribute to me). Robertson and I have recently completed an accurate evaluation of this effect ${ }^{12}$, and the results show that it too helps substantially to narrow the gap between the two methods of arriving at the ocean uptake of $\mathrm{CO}_{2}$.
A.W. 